\title{
Carbohydrate Metabolism.
}

THE breakdown of the carbohydrates found in the body, namely, glycogen and glucose, resulting in their final oxidation into carbon dioxide and water and the liberation of the energy stored in their molecules, takes place by stages, the details of which are still imperfectly known. It appears probable that a compound of glucose with phosphoric acid forms an intermediate stage in the conversion of glycogen into glucose, while lactic and pyruvic acids are intermediate products in the conversion of the glucose into carbon dioxide. In the case of striped muscle, the process, as far as the formation of lactic acid, is reversible (Emden, Meyerhof, etc.). Now although the monosaccharides formed in the course of digestion are absorbed and converted into glycogen in the liver, in the reverse process only glucose is produced, suggesting that this sugar has some peculiarity of structure which enables the cells of the body to utilise it, in preference to the others : enzymes are probably concerned in the process, and one of the characteristics of their action is their specificity.

Fresh light has been thrown on this subject by the work of Herring, Irvine and Macleod (Biochem. Jour., I924, vol. I8, p. I023), who have used insulin as a key to the structure which the sugar must possess if it is to be utilised. The insulin was injected into mice, and the efficiency of various sugars and other closely related substances in curing the convulsions thus produced determined. Glucose and mannose cured the animals completely, but fructose and galactose produced only a temporary improvement. Maltose, however, was nearly as efficient as glucose, but somewhat slower in its action. None of the other substances tried, including substituted reducing sugars and glucosidic compounds, had any effect. Analysis of the results shows that the sugar must possess the following formula,

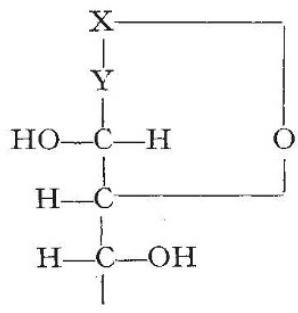

in which $\mathrm{X}$ or $\mathrm{Y}$ represents the reducing group. The cells of the body are, therefore, only fitted to deal with a sugar of this general type, all other carbohydrates being converted into it by their appropriate enzymes, either directly or after synthesis into glycogen.

Working on quite different lines, Kay and Robison and their co-workers have investigated the properties and functions of the compounds of glucose with phosphoric acid (Biochem. Jour., I924, vol. I8, pp. II33, II39, II52, II6I). Two of these compounds are described, a hexosemonophosphate and a hexosediphosphate, the phosphoric acid being combined with a different group of the sugar in each case. Both compounds are probably present in the blood. An investigation of the distribution in the body of the ferments which act upon them shows that the diphosphate is hydrolysed by an enzyme present chiefly in muscle and blood, while the monophosphate is acted on by an enzyme which can be extracted from bone and ossifying cartilage. In fact, it is suggested that the latter compound is the form in which phosphorus is supplied to the bone in ossification. The injection of insulin increases the amount of the organic phosphoric esters in the blood and muscles, and it is extremely probable that it is the hexosephosphates which are thus affected. In this case the fall in the blood sugar after insulin may be connected with the formation of these esters, since it appears that the sugar disappearing is not all oxidised, nor is it converted into fat (v. Burn and Dale, Jour. Physiol., I924, vol. 59, p. I64). The formation of a phosphoric ester would, however, be probably only a step in the reversible reaction, glycogen $\rightleftarrows$ glucose. The actual direction in which the reaction proceeds depends in all probability on the amount of glycogen in the body: in the well-fed animal, the glycogen present is broken down; in the diabetic organism, the reverse process occurs and glycogen appears in the liver.

The phosphorus for the synthesis of the ester comes in the first place from the inorganic phosphate of the blood, with the result that less is left available for excretion by the kidney in the urine. Sokhey and Allan (Biochem. Jour., I924, vol. I8, p. II70), confirming other investigators, have found that dogs after large doses of insulin excrete less inorganic phosphate in the urine during a period of six hours or so: the total output for the day is, however, definitely increased, suggesting that some of the newly formed esters are broken down later on. The nitrogen excretion is raised simultaneously with the fall in inorganic phosphate: possibly some of the phosphorus required comes from organic compounds which aiso contain nitrogen. Sugar per os, on the other hand, while diminishing the inorganic phosphorus of the blood and urine temporarily, does not cause any increased total output of this element in the 24 hours: at the same time it lowers the nitrogen excretion by taking the place of some of the protein previously oxidised. It appears then that, with excess of sugar in the blood, more of the esters are formed at the expense of the inorganic phosphorus, but that, although the subsequent oxidation is sufficient to reduce the nitrogen excretion by sparing the destruction of protein, it is not enough to raise appreciably the excretion of phosphorus in the urine; the sugar is probably oxidised directly without previous synthesis to glycogen. Insulin, however, causes a great increase in the formation of the esters, the phosphorus coming both from inorganic phosphates and also from bodies which contain nitrogen as well as phosphorus in their molecules. The glucose necessary is obtained from that circulating in the body fluids, but in the normal animal an additional source is provided by the reserve glycogen of the liver and muscles; the breakdown of the latter would produce an excess of free phosphorus, which would appear in the urine.

A somewhat different reaction is to be expected from feeding sugar and injecting insulin : glucose alone will not raise the total metabolism to any appreciable extent, while insulin, under certain conditions, will do so by increasing the carbohydrate oxidised, without at the same time reducing the protein broken down: in the normal animal, a limit is probably set to the amount of carbohydrate catabolised at any moment by the amount of insulin available. It seems justifiable to draw the general conclusion that insulin

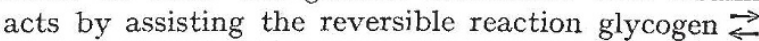
phosphoric ester $\rightleftarrows$ glucose, by causing the production of the ester; the direction in which the reaction proceeds thereafter depends on the amounts of the various interacting substances present at the moment. 